\title{
The new RNAi-based therapy for brain tumors
}

\author{
KATARZYNA ROLLE *, JAN BARCISZEWSKI \\ Institute of Bioorganic Chemistry, Polish Academy of Sciences, Poznań, Poland
}

\author{
US Patent \\ Patent No.: US 8,946,400 B2, \\ Title of invention: Sequence of dsRNA: ATN-RNA specific for tenascin-C \\ Inventors: Jan Barciszewski, Mirosława Barciszewska, Leszek Rychlewski, Eliza Wyszko, Katarzyna Rolle, Iwona \\ Gawrońska, Ryszard Żukiel, Stanisław Nowak \\ Assignees: Institute of Bioorganic Chemistry Polish Academy of Sciences Poznan, Poznan University of Medical \\ Sciences, Bioinfobank Sp. z o.o., Poznan \\ Date of patent: February 3, 2015 \\ Application number: US 12/375,916 \\ PCT number: PCT/PL2007/000054 \\ Application date: July 30, 2007 \\ Foreign application priority date: July 31, 2006 \\ Published also as: EP2121927A2, EP2121927B1, US20100076053, WO2008016317A2, WO2008016317A3
}

\begin{abstract}
The subject matter of this invention includes a sequence of double-stranded RNA: ATN-RNA, intervention using interference RNA intervention (iRNAi); the use of a sequence of double-stranded RNA: ATN-RNA, a method of treating (a) brain tumor and a method of inhibiting brain tumor cells which express tenascin, a kit for inhibiting cancer cell which expresses tenascin and a method for a kit preparation in a brain tumor therapy. Malignant gliomas preferentially express a number of surface markers that may be exploited as therapeutic targets, including tenascin-C, an extracellular matrix glycoprotein ubiquitously expressed by malignant gliomas, which probably contributes to the tumor cell adhesion, invasion, migration, and proliferation. For tenascin-C inhibition, the iRNAi approach has been applied.
\end{abstract}

\section{State of the art}

\section{Brain tumors}

The World Health Organization (WHO) has classified gliomas into heterogeneous group of tumors as follows: pilocytic astrocytomas (WHO I) with slow growth and rarely undergoing malignant transformation and three groups of diffusely infiltrative astrocytomas comprising diffuse astrocytomas (WHO II), anaplastic astrocytomas (WHO III), and glioblastoma (WHO IV).

As described in the WHO classification, malignant diffuse gliomas are comprised of astrocytic, oligodendroglial, and mixed oligoastrocytic neoplasm based solely on mor- phology and are further subdivided by tumor grade based on additional histologic features present in the tumor.

Glioblastoma multiforme (GBM), as indicated by the word "multiforme", displays a highly heterogeneous composition of cells and exhibits phenotypic heterogeneity. GBM is associated with histopathological features such as cellular polymorphism, atypia, substantial mitotic activity (with three - fivefold higher proliferative rates than grade III anaplastic astrocytoma), vascular thrombosis, and the most essential in terms of diagnosis - microvascular proliferation and necrosis. Other prominent features of GBM are regional heterogeneity, highly invasive growth, and diffuse infiltration of the surrounding brain.

\footnotetext{
* Corresponding author: Institute of Bioorganic Chemistry, PAS, Noskowskiego 12/14, 61-704 Poznań, Poland; e-mail: kbug@ibch.poznan.pl
} 
Patients with glioblastoma invariably fail to achieve long-term survival, which is thought to result from the acquired resistance to chemotherapeutics or radiation. Despite a broad approach to the treatment, the median patient survival is approximately 6-8 months although about $40 \%$ of patients with GBM die within 6 months after the diagnosis.

\section{Tenascin-C}

In many neoplasms, up-regulation of certain growth factors or their receptors or deregulation of intracellular signal transduction pathways represent key elements in the process of malignant transformation and progression of normal cells toward tumor cells, leading to uncontrolled proliferation and decreased apoptosis. Altered expression levels of certain genes play a pivotal role in several pathological conditions.

Malignant gliomas preferentially express a number of surface markers that may be exploited as therapeutic targets, including tenascin-C (TN-C), an extracellular matrix glycoprotein that is ubiquitously expressed by malignant gliomas, probably contributing to tumor cell adhesion, invasion, migration, and proliferation.

The high expression of TN-C in cancerous tissue and the in vitro activities strongly suggest that TN-C is a key determinant of the tumor stroma that is involved in the initiation of tumorigenesis and progression to metastasis.

The TN-C level correlates well with enhanced tumorigenesis and supports malignant transformation, uncontrolled proliferation, metastasis, angiogenesis and escaping from tumor immunosurveillance. We have also shown that TN-C correlates well with the WHO grade - the most malignant tumors, the more TN-C expressed, which gives the inverse correlation with prognosis and survival.

TN-C and its splice variant expression correlate with increased cell migration, tissue remodeling, angiogenesis, and local infiltration of normal tissues by tumor cells of various carcinomas. This is one of the main reasons why the outcome for primary central nervous system tumors, including glioblastoma multiforme (GBM), which is the most common and deadliest primary adult malignant brain tumor, remains unacceptable. Although standard treatment approaches to brain tumor can prolong life, they have little impact on survival. At the time of appearance and recurrence of the tumor, standard options are limited by the tumor location, size, and the overall condition of the patient. A few patients may be candidates for reoperation, but prolonged survival is rare with this approach alone.

As the failure of treatment is usually due to insufficient local control of the site of surgical resection, novel local therapies are still necessary. There is still a need to create a technology that might be used in the area of neoplastic brain infiltration that cannot be removed surgically, with no influence on intracranial pressure - volume relationships and with no local or general inflammatory response.

The development of more effective alternative treatments will be crucial to improve the survival of patients with these tumors. As most malignant gliomas are well known to be chemo- and radio-resistant due to the inhibition of an apoptotic pathway, the RNA interference (RNAi) may be suitable for their treatment.

\section{RNAi approach}

RNAi is a sequence-specific and conserved pathway in which double stranded RNA molecules (dsRNA) have been identified as mediators of functional gene silencing of specific genes in a variety of eukaryotic organisms. dsRNAs are processed into small RNAs 20-30 nucleotides long by the ribonuclease III (RNase III) enzyme Dicer.

The small RNAs are the specificity determinants of the pathway, assembling into the RNA-induced silencing complex (RISC), a multiprotein entity guided to complementary RNA, resulting in silencing of the message.

For the down-regulation of TN-C in brain tumor, we applied the iRNAi. After the surgery for patients with malignant gliomas, iRNAi with ATN-RNA is a promising local adjuvant therapy. It involves the direct delivery of the therapeutical dsRNA into the tumor tissue resected area, which induces RNA interference effects downstream of the application site, specifically down-regulating the synthesis of TN-C. This is the site - directed therapy that may improve local control and overall outcome for malignant glioma patients. A locally administered therapy offers the ability to achieve a greater effective concentration in the tumor vanity by bypassing the blood-brain barrier while limiting the potential systemic exposure to drug degradation. 
Patients suffering from brain tumor who qualified for surgery and molecular intervention were managed/treated at the Department of Neurosurgery and Neurotraumatology, University of Medical Sciences in Poznan. Before every surgical procedure, a precise tumor location was determined with computerized tomography (CT) or magnetic resonance imaging (MRI). A total of 46 patients with brain tumor were analyzed and diagnosed according to $\mathrm{WHO}$ criteria. Of those, 25 showed primary WHO IV, 7 WHO III, and 14 WHO II. After the surgical resection, RNAi was injected into the postoperative area. The efficacy of the ATN-RNA treatment was estimated on the basis of follow-up studies including general and neurological condition as well as neuroimaging studies with the CT and MRI measured for each two-month period after the treatment.

The patent claims:

- The subject of the invention is a sequence of a dsRNA: ATN-RNA, complementary to a/the fragment of the TN-C mRNA sequence at nucleotides 406 to 569. It forms a $164 \mathrm{nt}$ long two-stranded RNA (dsRNA). The dsRNA sequence is introduced into the cell and that from this piece any smaller fragments of 20-25 nt may form.

- The next subject of invention is an intervention using RNA interference (iRNAi), characterized in that ATN-RNA is introduced into a post-operative space, encompassing the conjunction of neurosurgical removal of a majority of tumor with the direct injection of mRNA into the remaining, inoperable tumor cells.

- The treatment is designed especially to human brain tumors (astrocytic brain tumor, such as glioblastoma multiforme, anaplastic astrocytoma, anaplastic oligoastrocytoma, or oligodendroglioma). An administration step is carried out by injecting a therapeutically effective amount of the sequence in an amount between 80 and $200 \mu \mathrm{g}$.

- The next subject of invention is a method for a kit preparation, wherein the kit is for use in inhibiting cancer cell that expresses TN-C. In this kit, ATN-
RNA is prepared by the transcription of ATN-DNA with T7/T3 polymerases on the plasmid harboring TN-C DNA cleaved with HindIII or EcoRI, further supplemented with $\mathrm{CaCl}_{2}$ and then delivered directly into surgically created resection cavity of patients with malignant brain tumor.

\section{Prospects}

The ATN-RNA sequence shows specificity in vitro and ex vivo toward TN-C mRNA. Direct delivery (injection) of the therapeutically dsRNA into the tumor tissue resected area induces of RNA interference effects and a specific down-regulation of the TN-C synthesis. The site-directed therapy offers a possibility to perform a local control and monitoring of the overall outcome for malignant glioma patients. To our knowledge, there are no clinical trials with an agent specifically designed to target the invasive cancer cells implemented for glioma treatment. While a highly invasive behavior of human glioma is widely recognized, most compounds are aimed at targeting "the tumor cells" rather than taking advantage of a specifically overexpressed "target" that would decrease the invasiveness of glioma cells. The development and proper application of an anti-invasive agent that would inhibit tumor invasion with no cytotoxic effect still holds a promise.

It seems that locally administered dsRNA (ATNRNA) therapy offers an ability to achieve a greater effective concentration in the tumor vanity by bypassing the blood-brain barrier while limiting the potential systemic exposure to drug degradation. ATN-RNA treatment can be combined with traditional therapy, but a great heterogeneity of GBM generates a necessity for further investigation of a "combined" glioma therapy, thus searching other therapeutical targets. Such a multitargeted approach involves a few glioma-specific molecular targets, such as, for example, TN-C, and seems to be the most promising treatment in the future not only for GBM, but also for other kinds of neoplasms. 